\title{
CO Isotopes in Planetary Nebulae
}

\author{
Dana S. Balser ${ }^{1}$, Joseph P. McMullin² ${ }^{2}$ \& T. L. Wilson ${ }^{3,4}$
}

\begin{abstract}
Standard stellar evolution theory is inconsistent with the observed isotopic carbon ratio, ${ }^{12} \mathrm{C} /{ }^{13} \mathrm{C}$, in evolved stars. This theory is also inconsistent with the ${ }^{3} \mathrm{He} / \mathrm{H}$ abundance ratios observed in Galactic $\mathrm{H}$ II regions, when combined with chemical evolution theory. These discrepancies have been attributed to an extra, non-standard mixing which further processes material during the RGB and should lower both the ${ }^{12} \mathrm{C} /{ }^{13} \mathrm{C}$ and ${ }^{3} \mathrm{He} / \mathrm{H}$ abundance ratios for stars with masses $\leq 2 M_{\odot}$. Measurements of isotopic ratios in planetary nebulae probe material which escapes the star to be further processed by future generations of stars.

We have measured the carbon isotopic abundance ratio, ${ }^{12} \mathrm{C} /{ }^{13} \mathrm{C}$, in 11 planetary nebulae (PNe) by observing the $\mathrm{J}=2 \rightarrow 1$ and $\mathrm{J}=3 \rightarrow 2$ millimeter transitions of ${ }^{12} \mathrm{CO}$ and ${ }^{13} \mathrm{CO}$ in molecular clouds associated with the PNe. A large velocity gradient (LVG) model has been used to determine the physical conditions for each $\mathrm{PNe}$ where both transitions have been detected. We detect both ${ }^{12} \mathrm{CO}$ and ${ }^{13} \mathrm{CO}$ in 9 PNe. If ${ }^{12} \mathrm{CO} /{ }^{13} \mathrm{CO}={ }^{12} \mathrm{C} /{ }^{13} \mathrm{C}$, the range of ${ }^{12} \mathrm{C} /{ }^{13} \mathrm{C}$ is $2.2-31$. Our results support theories which include some form of extra mixing.
\end{abstract}

Subject headings: ISM: abundances — ISM: molecules — planetary nebulae: general - radio lines: ISM

\section{Introduction}

Measuring isotopic abundance ratios is one method of probing how stars of various masses process material and how the Galaxy redistributes this material over time - that is, stellar and Galactic chemical evolution. Some of the light elements $\mathrm{H},{ }^{2} \mathrm{H},{ }^{3} \mathrm{He},{ }^{4} \mathrm{He}$, and ${ }^{7} \mathrm{Li}$, were produced during the era of primordial nucleosynthesis, so these ratios provide

\footnotetext{
${ }^{1}$ National Radio Astronomy Observatory, P.O. Box 2, Green Bank WV 24944

${ }^{2}$ National Radio Astronomy Observatory, 520 Edgemont Road, Charlottesville, VA 22903

${ }^{3}$ SMTO, Steward Observatory, University of Arizona, Tucson, AZ 85720

${ }^{4}$ Max-Planck-Institut für Radioastronomie, 53121 Bonn, Germany
} 
additional constraints for Big Bang nucleosynthesis. H II regions or molecular clouds are examples of zero-age objects which are young relative to the age of the Galaxy. Therefore these abundance ratios chronicle the results of billions of years of Galactic chemical evolution. Observations of isotopic ratios in stars and planetary nebulae (PNe) measure locally how stars influence chemical evolution. In particular, PNe probe material which has been ejected from low-mass $\left(M \leq 2 M_{\odot}\right)$ to intermediate-mass $\left(M \sim 2-5 M_{\odot}\right)$ stars to be further processed by future generations.

Measurements of ${ }^{3} \mathrm{He}$ in the Galaxy have led to what has been called "The ${ }^{3} \mathrm{He}$ Problem" (Galli et al. 1997). Standard stellar evolution theory predicts that ${ }^{3} \mathrm{He}$ is produced in significant quantities by stars of $1-2 M_{\odot}$ and that PNe with progenitors in that mass range should have ${ }^{3} \mathrm{He} / \mathrm{H} \sim$ several $\times 10^{-4}$ (Iben 1967; Rood 1972; Vassiliadis \& Wood 1993; Weiss et al. 1996). (All abundance ratios will be by number unless otherwise noted.) Galactic chemical evolution models which integrate over a range of initial masses predict that ${ }^{3} \mathrm{He}$ is produced in prodigious amounts (Rood et al. 1976; Dearborn et al. 1986). A survey of 6 PNe produced a detection of ${ }^{3} \mathrm{He}$ in NGC 3242 (Rood, Bania, \& Wilson 1992; Balser, Rood, \& Bania 1999) and probable detections in two other sources (Balser et al. 1997) with ${ }^{3} \mathrm{He} / \mathrm{H} \sim 10^{-4}-10^{-3}$ as predicted by standard stellar evolution models. This PNe sample was highly biased, however, and was selected to maximize the likelihood of a detection. Observations of ${ }^{3} \mathrm{He}$ in protosolar material (Geiss 1993), the local interstellar medium (Gloeckler \& Geiss 1996), and Galactic H II regions (Balser et al. 1999) all indicate a ${ }^{3} \mathrm{He} / \mathrm{H}$ abundance ratio of $\sim 2 \times 10^{-5}$. Although $\mathrm{H}$ II regions are expected to be zero-age objects there is no evidence for any stellar ${ }^{3} \mathrm{He}$ enrichment during the last $4.5 \mathrm{Gyr}$ (Bania et al. 2002). The observed abundances of ${ }^{3} \mathrm{He}$ throughout the Galaxy when combined with the observations that some stars do indeed produce ${ }^{3} \mathrm{He}$ strongly disagree with standard chemical evolution models (Steigman \& Tosi 1992; Vangioni-Flam et al. 1994; Steigman \& Tosi 1995; Dearborn et al. 1996; Prantzos 1996; Fields 1996; Scully et al. 1996; Galli et al. 1997).

Anomalies in the ${ }^{12} \mathrm{C} /{ }^{13} \mathrm{C}$ abundance ratio in red giant stars has stimulated studies of non-standard stellar evolution models (Charbonnel 1994, 1995; Wasserburg et al. 1995; Weiss et al. 1996; Sackmann \& Boothroyd 1999; Boothroyd \& Sackmann 1999). All of these models include some form of extra mixing which transports material in the convective envelope to lower regions which are hot enough for further nuclear processing and then transports it back up into the convective envelope. The extra processing that occurs is called cool bottom processing (CBP).

During the first dredge-up on the red giant branch (RGB), the convective envelope reaches regions where ${ }^{12} \mathrm{C}$ had previously been processed into ${ }^{13} \mathrm{C}$ and ${ }^{14} \mathrm{~N}$; thus we expect 
${ }^{12} \mathrm{C} /{ }^{13} \mathrm{C}$ to decrease from $\sim 90$ to 25 for both low-mass and intermediate-mass stars (Iben 1964; Iben \& Renzini 1984). Observations of carbon in the surfaces of red giant stars reveal lower ${ }^{12} \mathrm{C} /{ }^{13} \mathrm{C}$ ratios $(5-20)$ for stars below $2 M_{\odot}$ than theoretically expected (e.g., Gilroy 1989). Studies which measure the evolution of the surface ${ }^{12} \mathrm{C} /{ }^{13} \mathrm{C}$ as a star ascends the RGB in M67 (Gilroy \& Brown 1991) and in field stars (Charbonnel et al. 1998) indicate that additional processing occurs between the base and tip of the RGB. This has been attributed to extra mixing below the convective envelope.

During the asymptotic giant branch (AGB) the theoretical abundances depend on the interplay between dredge-up events, mass loss, hot bottom burning, and non-standard mixing (CBP). During the third dredge-up on the AGB significant pollution of ${ }^{12} \mathrm{C}$ will increase the ${ }^{12} \mathrm{C} /{ }^{13} \mathrm{C}$ ratio if hot bottom burning does not take place (Forestini \& Charbonnel 1997). In low-mass stars, ${ }^{12} \mathrm{C} /{ }^{13} \mathrm{C}$ may decrease to $\sim 4$ if $\mathrm{AGB}$ cool bottom processing occurs (Wasserburg et al. 1995). For AGB stars more massive than $\sim 4 M_{\odot}$, hot bottom burning drives the ${ }^{12} \mathrm{C} /{ }^{13} \mathrm{C}$ towards its equilibrium value of 3.5 (Frost et al. 1998).

Observations of abundances in PNe measure the cumulative effects of different mixing and nuclear processing events during the entire evolution of their progenitors. In general the abundance of ${ }^{3} \mathrm{He}$ should follow the ${ }^{12} \mathrm{C} /{ }^{13} \mathrm{C}$ ratio; that is, if ${ }^{3} \mathrm{He}$ is destroyed then the ${ }^{12} \mathrm{C} /{ }^{13} \mathrm{C}$ ratio should decrease. Therefore given the current observational challenges of detecting ${ }^{3} \mathrm{He}$ in $\mathrm{PNe}$, an alternative approach is to measure ${ }^{12} \mathrm{C} /{ }^{13} \mathrm{C}$.

There have been several studies of ${ }^{12} \mathrm{C} /{ }^{13} \mathrm{C}$ in the molecular gas in PNe using millimeter transitions of CO (Bachiller et al. 1989; Jaminet et al. 1991; Cox et al. 1992; Bachiller et al. 1997; Palla et al. 2000). In these studies it is assumed that the ${ }^{12} \mathrm{C} /{ }^{13} \mathrm{C}$ ratios measured in molecular clouds near the PN refer to the abundances in the PN itself. Alternatively, Clegg et al. (1997) and Miskey et al. (2000) have used the ${ }^{13} \mathrm{C}_{1 / 2}^{3} \mathrm{P}_{0}^{o}-{ }_{1 / 2}^{1} \mathrm{~S}_{0}$ transition at $1909.597 \AA$ to measure the ${ }^{12} \mathrm{C} /{ }^{13} \mathrm{C}$ ratio in several PNe. From these studies the measured ${ }^{12} \mathrm{C} /{ }^{13} \mathrm{C}$ abundance ratios are between $\sim 10$ to 40. Palla et al. (2000; hereafter PBSTG) concluded that in the majority of $\mathrm{PNe}$ the ${ }^{12} \mathrm{C} /{ }^{13} \mathrm{C}$ ratio is lower than expected from standard stellar models and are consistent with non-standard mixing models which incorporate CBP.

In this paper we present new observations of ${ }^{12} \mathrm{CO}$ and ${ }^{13} \mathrm{CO}$ which enlarge the $\mathrm{PN}$ sample. Usually isotope ratios are estimated using optically thin lines, however, since the $\mathrm{CO}$ intensities are small, and since the ${ }^{16} \mathrm{O} /{ }^{18} \mathrm{O}$ ratios may vary, the ${ }^{12} \mathrm{CO}$ and ${ }^{13} \mathrm{CO}$ lines are used to deduce the ${ }^{12} \mathrm{C} /{ }^{13} \mathrm{C}$ ratio. Because of the resulting uncertainty in opacity, it is important that at least two transitions of $\mathrm{CO}$ be observed to constrain radiative models used to calculate the ${ }^{12} \mathrm{C} /{ }^{13} \mathrm{C}$ abundance ratio. The observations are discussed in $\S 2$ and the general results are in $\S 3$. In $\S 4$ the ${ }^{12} \mathrm{C} /{ }^{13} \mathrm{C}$ abundance ratios are calculated using the assumption that the optical depths of both ${ }^{13} \mathrm{CO}$ and ${ }^{12} \mathrm{CO}$ are small and that the transitions 
are themalized. More sophisticated methods are explored in $\S 5$. In $\S 6$ the implications with respect to stellar and Galactic evolution are discussed. A summary is given in $\S 7$.

\section{Observations}

The observed sample of PNe are listed in Table 1. The PNe were selected from the CO survey of Huggins \& Healy (1989) based on the estimated intensity of ${ }^{13} \mathrm{CO}$ for an expected ${ }^{12} \mathrm{CO} /{ }^{13} \mathrm{CO}$ ratio of 25 . Listed are the source name, the Galactic coordinates rounded to the nearest degree, the equatorial coordinates, the observed offset position, the LSR velocity, the angular size, the progenitor main sequence mass, and the morphology. The progenitor masses, $M_{\mathrm{ms}}$, are taken from PBSTG for objects in common or determined by first using the central star masses determined by Górny, Stasińska, \& Tylenda (1997) or Stasińska, Górny, \& Tylenda (1997) and the initial-final mass relation of Herwig (1996). The PNe morphology is defined as round (R), elliptical (E), or bipolar (B) (Balick 1987; Manchado et al. 1996; Huggins et al. 1996).

An observing log is summarized in Table 2 which includes the spectral transitions, the telescope's half-power beam-width, and the velocity resolution. Observations of the ${ }^{12} \mathrm{CO}$ and ${ }^{13} \mathrm{CO}(\mathrm{J}=2 \rightarrow 1)$ rotational transitions were made with the National Radio Astronomy Observatory $^{5} 12 \mathrm{~m}$ telescope on 1997 May and 1997 December. The 200-300 GHz dualpolarization SIS receiver was employed. Both the filter-bank and hybrid spectrometers were used simultaneously and were configured to provide spectral resolutions of $1 \mathrm{MHz}$ and $2 \mathrm{MHz}$ per channel which produce a velocity resolution of $1.4 \mathrm{~km} \mathrm{sec}^{-1}$ and $2.7 \mathrm{~km} \mathrm{sec}^{-1}$ per channel, respectively. The spectra were obtained by beam switching plus position switching (BSP). The reference position (OFF) was typically 4 arcmin from the source position (ON). The pointing should be accurate to within $\sim 5$ arcsec. The data were calibrated on-line to the radiation temperature $\left(\mathrm{T}_{\mathrm{R}}^{*}\right)$ scale which corrects for atmospheric attenuation, radiative loss, and rearward and forward scattering and spillover (Kutner \& Ulich 1981). In this paper we report all intensities in units of the main beam brightness temperature, $\mathrm{T}_{\mathrm{mb}}$, using an efficiency of $\eta_{\mathrm{m}}^{*}=0.5$ which converts the radiation temperature to the main beam brightness temperature (Mangum 1999).

Observations of the ${ }^{12} \mathrm{CO}$ and ${ }^{13} \mathrm{CO}(\mathrm{J}=3 \rightarrow 2)$ transitions were made with the Henrich Hertz Telescope $(\mathrm{HHT})^{6}$ during February 1998 and May 1998. The facility $345 \mathrm{GHz}$

\footnotetext{
${ }^{5}$ The National Radio Astronomy Observatory is a facility of the National Science Foundation operated under cooperative agreement by Associated Universities, Inc.

${ }^{6}$ The Heinrich Hertz Telescope is operated by the Submillimeter Telescope Observatory on behalf of
} 
dual-polarization SIS receiver was employed, although because of technical difficulties only one channel was used. The spectrometers consisted of a $1 \mathrm{GHz}, 1024$-channel AOS and a $250 \mathrm{MHz}$, 1024-channel AOS with spectral resolutions of $1.0 \mathrm{MHz}\left(0.87 \mathrm{~km} \mathrm{sec}^{-1}\right)$ and $0.40 \mathrm{MHz}\left(0.35 \mathrm{~km} \mathrm{sec}^{-1}\right)$ per channel, respectively. Beam switching was used to produce the spectra using a procedure called WSWITCH with a reference offset from the source by 4 arcmin. (This procedure is effectively identical to BSP at the $12 \mathrm{~m}$.) The pointing model was determined to be accurate to within 1-2 arcsec for most locations in the sky. The data were calibrated on-line to the $\mathrm{T}_{\mathrm{A}}^{\prime}$ temperature scale which corrects for atmospheric attenuation. The line intensities were scaled to main beam brightness temperatures using several standard calibration sources ( $\chi$ Cyg, R Aql, and IRC+10216). These intensities are consistent with an assumed efficiency of $\eta_{\mathrm{mb}}=0.55$, if the mixer sideband ratios are equal. We estimate that the uncertainties in the intensity scale are between 15-20\% (Wilson et al. 2001).

\section{Results}

The results are summarized in Table 3. Listed are the source name, the transition, the peak intensity $\left(T_{\text {peak }}\right)$, the root-mean-squared $(R M S)$ variations in the spectral baseline, the velocity of the centroid ( $V_{\text {centroid }}$ ), the full-width half-maximum of the linewidth based on a single-component Gaussian fit $(\Delta V)$, and the integrated intensity $(I)$. Only $\Delta V$ is based on a Gaussian model. The data were analyzed independently using the spectral line analysis software CLASS, UniPops, and COMB. The majority of the sources were detected in both the ${ }^{12} \mathrm{CO}$ and ${ }^{13} \mathrm{CO} \mathrm{J}=2 \rightarrow 1$ transitions. Four objects were also detected in the $\mathrm{J}=3 \rightarrow 2$ transitions which require higher excitation.

Figure 1 summarizes the spectra taken with both telescopes. The first two columns correspond to the NRAO $12 \mathrm{~m} \mathrm{~J}=2 \rightarrow 1$ transitions while the last two columns correspond to the HHT $10 \mathrm{~m} \mathrm{~J}=3 \rightarrow 2$ transitions. The spectra have been shifted in velocity to align the profiles relative to the LSR velocity in Table 1. For several sources there is evidence for complicated velocity structure (e.g. multiple peaks, asymmetries, etc.). In these cases this structure is being averaged over the telescope's beam and thus our analysis refers to the bulk properties of the molecular emitting gas.

For PNe observed at both transitions we measure the $\mathrm{J}=3 \rightarrow 2$ transition to be significantly more intense than the $\mathrm{J}=2 \rightarrow 1$ transition. This effect is expected based on solely the radiative transfer in gas with physical conditions characteristic of PNe.

Steward Observatory and the Max-Planck-Institut für Radioastronomie. 
In Figure 2, the radiation temperature is plotted versus the upper $\mathrm{J}$ level rotational transition for $\mathrm{CO}$ over a range of densities. Across a broad range of physical conditions, the $\mathrm{J}=3 \rightarrow 2$ transition produces the brightest intensity; only in the lowest volume density-column density models is the $\mathrm{J}=2 \rightarrow 1$ intensity higher. The generally better coupling between smaller beams (obtained at higher frequencies) and PN structure also suggests that CO searches in PNe may be better carried out in the sub-millimeter regime; atmospheric and hardware limitations may mitigate this effect.

\section{4. ${ }^{12} \mathrm{C} /{ }^{13} \mathrm{C}$ Abundance Ratios}

The derived ${ }^{12} \mathrm{C} /{ }^{13} \mathrm{C}$ ratios for our PN sample are listed in Table 4 . In columns 3 and 4 the ${ }^{12} \mathrm{C} /{ }^{13} \mathrm{C}$ ratios are shown for the $\mathrm{J}=2 \rightarrow 1$ and $\mathrm{J}=3 \rightarrow 2$ transitions, respectively. Values based on the integrated intensities are expected to be more accurate, but values based on the peak intensities are also given (in parentheses). The ${ }^{12} \mathrm{CO} /{ }^{13} \mathrm{CO}$ abundance ratios are believed to be a direct measure of the ${ }^{12} \mathrm{C} /{ }^{13} \mathrm{C}$ ratio; therefore we have assumed that ${ }^{12} \mathrm{C} /{ }^{13} \mathrm{C}$ $={ }^{12} \mathrm{CO} /{ }^{13} \mathrm{CO}$. Chemical fractionation will lower the observed ${ }^{12} \mathrm{CO} /{ }^{13} \mathrm{CO}$ ratios, but this effect should be reduced by the observed high temperatures in the molecular gas $(\sim 25-60$ $\mathrm{K}$, Bachiller et al. 1997). Selective photo-dissociation of ${ }^{13} \mathrm{CO}$ causes an increase in the ${ }^{12} \mathrm{CO} /{ }^{13} \mathrm{CO}$ ratio but this will be counterbalanced by the charge exchange reaction ${ }^{13} \mathrm{C}^{+}+$ ${ }^{12} \mathrm{CO} \rightarrow{ }^{12} \mathrm{C}^{+}+{ }^{13} \mathrm{CO}$ (Likkel et al. 1988).

The range of ${ }^{12} \mathrm{C} /{ }^{13} \mathrm{C}$ ratios are lower than the values predicted by standard stellar models. About half of the objects observed in this study were also included in the sample of PBSTG. In most cases our ratios agree with the PBSTG values shown in the last column in Table 4. The peak main brightness temperatures for our observations are lower in all cases, as expected for the larger beam in our study. However the intensity is not proportional to the beam areas.

These ${ }^{12} \mathrm{C} /{ }^{13} \mathrm{C}$ ratios were calculated under the assumptions that the filling fraction for the gas is the same for both isotopomers, both rotational transitions are thermalized to the same temperature, and that the emission lines are optically thin. The first assumption seems reasonable based on the spectra shown in Figure 1. A comparison of the different transitions reveals nearly identical $V_{\mathrm{LSR}}$ and line shape characteristics, indicating that similar volumes of gas are sampled by the two transitions. The excitation characteristics of the low-J transitions of $\mathrm{CO}$ also supports the assumption of a thermalized, uniform temperature. The small dipole moment of $\mathrm{CO}$ results in a low critical density for rotational excitation; thus most PNe have densities high enough for significant CO excitation. The effect of line opacity in CO may be accounted for in multi-transition models of radiation transport. 


\section{Large Velocity Gradient Models}

In order to better interpret our results, the radiative transfer in the $\mathrm{PNe}$ is examined through the use of the Sobelev or Large Velocity Gradient (LVG) approximation (Sobolev $1958,1960)$. This model provides a numerical solution to the coupled equations of radiative transfer and statistical equilibrium for the rotational transitions of CO (see e.g. Leung \& Liszt 1976). In particular, we used the RAD package which is a part of the MIRIAD ${ }^{7}$ software package (Mundy 1992). Only the four PNe for which we have measured multiple transitions of ${ }^{12} \mathrm{CO}$ and ${ }^{13} \mathrm{CO}$ emission are considered. For each source a two-dimensional grid composed of volume density $(n)$ and column density $(N)$, over a range of physical conditions relevant to molecular gas in PNe, is generated. We adopt a kinetic temperature $T_{\mathrm{k}}=60 \mathrm{~K}$. This value is towards the high end of temperatures suggested by Bachiller et al. (1997); however, the higher J transitions of CO typically trace warmer regions and the LVG grids are consistent with $T_{\mathrm{k}}=60 \mathrm{~K}$. Data taken with the $\operatorname{HHT}(\mathrm{J}=3 \rightarrow 2)$ have been spatially smoothed to match the resolution of the $12 \mathrm{~m}(\mathrm{~J}=2 \rightarrow 1)$ data. We assume that the emission fills the beam for both pairs of transitions, or has a similar beam filling fraction. We obtained $\chi^{2}$ values from a comparison of the data to the model grids as a measure of the quality of the fits.

The derived ${ }^{12} \mathrm{C} /{ }^{13} \mathrm{C}$ ratios are listed in column 5 of Table 4 . The quoted uncertainties were determined by propagating the measured error in the integrated intensities from Table 3. These statistical uncertainties may be much smaller than the systematic uncertainties. For the PNe for which there only exists $\mathrm{J}=2 \rightarrow 1$ detections the integrated intensity ratios are adopted. Where the line shape is parabolic the line is optically thick so the ${ }^{12} \mathrm{C} /{ }^{13} \mathrm{C}$ ratios are cited as lower limits. For PNe where we have detected both the $\mathrm{J}=2 \rightarrow 1$ and $\mathrm{J}=3 \rightarrow 2$ transitions an LVG analysis is performed and a ${ }^{12} \mathrm{C} /{ }^{13} \mathrm{C}$ ratio determined. Below we discuss each of these sources in more detail.

AFGL 618 - Using ${ }^{13} \mathrm{CO}$ we derive $n=6.8 \times 10^{4} \mathrm{~cm}^{-3}$ and $N=6.8 \times 10^{15} \mathrm{~cm}^{-2}$. Using these values, we obtain $N=3.2 \times 10^{16} \mathrm{~cm}^{-2}$ for ${ }^{12} \mathrm{CO}$, yielding a ratio of ${ }^{12} \mathrm{CO} /{ }^{13} \mathrm{CO} \sim 5$. Overall this value is consistent with the observations. This model overproduces the $\mathrm{J}=2 \rightarrow 1$ emission by about $20 \%$ and the best fit density to the $\mathrm{J}=2 \rightarrow 1$ is a factor of $\sim 2$ lower than that derived from the ${ }^{13} \mathrm{CO}$. This may suggest that the observed ${ }^{12} \mathrm{CO} /{ }^{13} \mathrm{CO}$ ratio is a lower limit, since the ${ }^{12} \mathrm{CO}$ and ${ }^{13} \mathrm{CO}$ emission regions are not identical, or that density, temperature, and abundance gradients exist which are not isolated in velocity space. Although the derived opacity in the $\mathrm{J}=3 \rightarrow 2$ and $\mathrm{J}=2 \rightarrow 1$ lines from the LVG grids is low, the line shape is strongly suggestive of high opacity. We therefore cautiously cite the adopted ratio as a lower limit

\footnotetext{
${ }^{7}$ Multichannel Image Reconstruction, Image Analysis, and Display software developed by BerkeleyIllinois-Maryland Association.
} 
only.

M1-16 The model for M1-16 produces $n=6.8 \times 10^{4} \mathrm{~cm}^{-3}$ and $N=6.8 \times 10^{15} \mathrm{~cm}^{-2}$ for ${ }^{13} \mathrm{CO}$, and $N=1.5 \times 10^{16} \mathrm{~cm}^{-2}$ for ${ }^{12} \mathrm{CO}$, yielding ${ }^{12} \mathrm{CO} /{ }^{13} \mathrm{CO}=2.2$, very similar to that derived from both the peak and integrated intensities. Although this value is very low, the model suggests that the opacity remains low for both transitions (e.g., $\tau<0.05$ for ${ }^{12} \mathrm{CO}$ ). PBSTG consider this source to be a lower limit, however, presumably because of high opacity. Examining the shape of the spectrum, the profile seems most like the classical flat topped structure of an optically thin, unresolved shell. Therefore we adopt our model-derived value for the ratio in this source.

IRAS 21282+5050 - Using ${ }^{13} \mathrm{CO}$ we obtain $n=3.2 \times 10^{5} \mathrm{~cm}^{-3}$ and $N=1.0 \times 10^{15} \mathrm{~cm}^{-2}$. This produces a column density of $3.2 \times 10^{16} \mathrm{~cm}^{-2}$ and a ratio of ${ }^{12} \mathrm{CO} /{ }^{13} \mathrm{CO}=32$. The volume density for IRAS $21282+5050$ is larger than that obtained from the other sources, perhaps indicative of its youth. The shape of its spectral profile is difficult to discern given the signal-noise in our data. For ${ }^{12} \mathrm{CO}$ in the $\mathrm{J}=3 \rightarrow 2$ line the opacity should be higher but this line seems more parabolic than flat topped. Moreover, the values obtained from the ratios of the integrated intensities and peak intensities are not consistent, most likely due to the low signal-to-noise ratio in the ${ }^{13} \mathrm{CO}$ data. Due to these concerns, we adopt ${ }^{12} \mathrm{CO} /{ }^{13} \mathrm{CO}$ $=32$ as a lower limit.

NGC 7293 - Numerous models were run for NGC 7293 without obtaining any reasonable fits to the individual ${ }^{12} \mathrm{CO}$ or ${ }^{13} \mathrm{CO}$ data. In addition, the derived physical characteristics for ${ }^{13} \mathrm{CO}$ were highly inconsistent with those from ${ }^{12} \mathrm{CO}$. Since NGC 7293 is an evolved $\mathrm{PN}$, the simplest explanation for these results is that structure in the CO line emitting gas couples differently in the two beams, so the $\mathrm{J}=2 \rightarrow 1$ and $\mathrm{J}=3 \rightarrow 2$ regions are distinct and different. The emission profiles from the lines do not suggest a high opacity, however. Since the $\mathrm{J}=3 \rightarrow 2{ }^{13} \mathrm{CO}$ transition only has a signal-to-noise ratio of $\sim 4$ we adopt the $\mathrm{J}=2 \rightarrow 1$ ratio.

\section{Discussion}

The carbon isotopic ratio, ${ }^{12} \mathrm{C} /{ }^{13} \mathrm{C}$, along with other elements are processed during different stages of stellar evolution such as the red giant branch (RGB), the asymptotic

giant branch (AGB), and the planetary nebula $(\mathrm{PN})$. Observations have been made during these different stages of evolution which can be used to constrain stellar evolution theory.

Observations of anomalous ${ }^{12} \mathrm{C} /{ }^{13} \mathrm{C}$ abundance ratios in RGB field stars (Tomkin, Lambert, \& Luck 1975; Tomkin, Luck, \& Lambert 1976) and open clusters (Gilroy 1989) 
have stimulated theoretical studies which introduce an additional mixing process to reduce ${ }^{12} \mathrm{C} /{ }^{13} \mathrm{C}$ in stars (Sweigart \& Mengel 1979; Charbonnel 1994, 1995; Wasserburg et al. 1995). A detailed study of $\mathrm{M} 67$ revealed that the anomalously low ${ }^{12} \mathrm{C} /{ }^{13} \mathrm{C}$ ratios occurred only for stars at the tip of the RGB (Gilroy \& Brown 1991). Reanalysis of 191 field and cluster giants stars using HIPPARCOS parallaxes has determined that $96 \%$ of evolved stars, objects which have passed the luminosity bump, have ${ }^{12} \mathrm{C} /{ }^{13} \mathrm{C}$ ratios lower than standard models predict (Charbonnel \& Do Nascimento 1998). Gratton et al. (2000) have measured ${ }^{12} \mathrm{C} /{ }^{13} \mathrm{C}$ in 62 field metal-poor stars, including 43 additional stars from the literature. They determine two distinct mixing episodes. The first dredge-up which produces ${ }^{12} \mathrm{C} /{ }^{13} \mathrm{C}$ ratios consistent with standard models $\left({ }^{12} \mathrm{C} /{ }^{13} \mathrm{C}=20-30\right)$; and a second mixing episode occurring after the luminosity bump, when the molecular weight barrier is removed, where ${ }^{12} \mathrm{C} /{ }^{13} \mathrm{C}$ is reduced to $6-10$. These results are consistent with the theoretical models which predict extra mixing (Charbonnel 1994, 1995).

Additional processing of carbon and other elements occurs on the AGB. Observations have been made of ${ }^{12} \mathrm{C} /{ }^{13} \mathrm{C}$ in $\mathrm{AGB}$ stars using mm-wave, optical, and IR spectroscopy. CO mm-wave observations of the circumstellar envelops of carbon and oxygen-rich AGB stars yield ${ }^{12} \mathrm{C} /{ }^{13} \mathrm{C}$ values between $\sim 10-50$ (Wannier \& Sahai 1987; Kahane \& Jura 1994; Greaves \& Holland 1997). Evidence for some deep mixing process is suggested from an optical study of carbon stars where Li-rich objects typically have low $(<15){ }^{12} \mathrm{C} /{ }^{13} \mathrm{C}$ ratios (Abia \& Isern 1997). IR spectroscopy of cool carbon stars produces ${ }^{12} \mathrm{C} /{ }^{13} \mathrm{C}$ ratios between $30-70$ with a few very low ratios, ${ }^{12} \mathrm{C} /{ }^{13} \mathrm{C} \sim 4$ (Lambert et al. 1986). The high ${ }^{12} \mathrm{C} /{ }^{13} \mathrm{C}$ values are expected from third dredge-up of ${ }^{12} \mathrm{C}$, while depending on the mass of the star the very low ${ }^{12} \mathrm{C} /{ }^{13} \mathrm{C}$ ratios could be explained by hot bottom burning or by deep mixing. In contrast Ohnaka \& Tsuji (1996) determine ${ }^{12} \mathrm{C} /{ }^{13} \mathrm{C}$ ratios that are $2-3$ times smaller than Lambert et al. even though there is a significant overlap in the stars observed. There have been heated discussions about the analysis of spectra using the iso-intensity method (de Laverny \& Gustafsson 1998; Ohnaka \& Tsuji 1998; de Laverny \& Gustafsson 1999; Ohnaka, Tsuji \& Aoki 2000). Nevertheless, it appears that at least some objects do have reduced ${ }^{12} \mathrm{C} /{ }^{13} \mathrm{C}$ ratios compared to that expected from standard models and that some extra mixing mechanism is at work.

Observations of ${ }^{12} \mathrm{C} /{ }^{13} \mathrm{C}$ in $\mathrm{PNe}$ measure how carbon has been processed through both the RGB and AGB phases. Because this processed material escapes into the interstellar medium these ${ }^{12} \mathrm{C} /{ }^{13} \mathrm{C}$ ratios can be used to constrain Galactic chemical evolution models. Figure 3 summarizes the current status of ${ }^{12} \mathrm{C} /{ }^{13} \mathrm{C}$ abundance ratios in PNe. Plotted are the determined ${ }^{12} \mathrm{C} /{ }^{13} \mathrm{C}$ ratios versus the estimated main sequence progenitor mass $\left(M_{\mathrm{ms}}\right)$. Only PNe for which progenitor masses could be found are shown. The results from other CO observations are taken from PBSTG and displayed as triangles. Measurements using the 
CIII] transition are shown as stars. Notice that there are five PNe that we have in common with PBSTG. These objects are indicated by open triangles.

For clarity only the (statistical) uncertainties in the ${ }^{12} \mathrm{C} /{ }^{13} \mathrm{C}$ ratio are shown in Figure 3. The uncertainties in the derived progenitor masses are significant and are mostly due to estimates of the PN distance and calculations of the progenitor mass from the current central star mass using some initial mass-final mass relationship. These uncertainties can be as large as $50 \%$ (e.g, Galli et al. 1997; PBSTG). Therefore care must be used when interpreting these results. The error bars in Figure 3 for ${ }^{12} \mathrm{C} /{ }^{13} \mathrm{C}$ ratios are based on the uncertainties in the measured integrated intensity for each transition. Note that the systematic errors may be much larger than these, in some cases.

Our results are consistent with PBSTG with the exception of NGC6720 where our ${ }^{12} \mathrm{C} /{ }^{13} \mathrm{C}$ ratio is a factor of two smaller. However, the signal-to-noise ratio for the ${ }^{13} \mathrm{CO}$ $\mathrm{J}=2 \rightarrow 1$ transition is only 5 and we do not have multiple transitions for this $\mathrm{PN}$. Therefore the ${ }^{12} \mathrm{CO}$ transition may be optically thick and our ${ }^{12} \mathrm{C} /{ }^{13} \mathrm{C}$ value a lower limit. However from the line shapes these data are consistent with optically thin emission.

The ${ }^{12} \mathrm{C} /{ }^{13} \mathrm{C}$ ratios range between $2.2-31$ with progenitor masses from $\sim 1-4 M_{\odot}$. This indicates that at least some stars do undergo some non-standard processing such as cool bottom processing (CBP). Because of the large uncertainties in determining the progenitor mass it is difficult to make any strong statements about the relationship of ${ }^{12} \mathrm{C} /{ }^{13} \mathrm{C}$ with $M_{\mathrm{ms}}$. Since CBP is expected to operate only for stars with $M_{\mathrm{ms}}<2 M_{\odot}$ the ${ }^{12} \mathrm{C} /{ }^{13} \mathrm{C}$ ratios should be larger for stars above this mass. Above $4 M_{\odot}$ hot bottom burning can drive the ${ }^{12} \mathrm{C} /{ }^{13} \mathrm{C}$ ratio towards it equilibrium value of 3.5 (Frost et al. 1998). The low ${ }^{12} \mathrm{C} /{ }^{13} \mathrm{C}$ values between 2-4 $M_{\odot}$ might indicate further processing on the AGB or that we have underestimated the ${ }^{12} \mathrm{C} /{ }^{13} \mathrm{C}$ ratios. It is interesting to note that the lowest ${ }^{12} \mathrm{C} /{ }^{13} \mathrm{C}$ values are classified as bipolar PNe. Bipolar PNe tend to lie closer to the Galactic plane with a wider distribution of central star masses and have higher N/O ratios (Górny, Stasińska, \& Tylenda 1997)

Since most of the PNe in our sample have only been detected in one transition we do not have sufficient constraints to determine the $\mathrm{CO}$ line opacity. If the ${ }^{12} \mathrm{CO}$ line is optically thick then the ${ }^{12} \mathrm{C} /{ }^{13} \mathrm{C}$ values will be underestimated and the points in Figure 3 must be increased. Clearly more ${ }^{12} \mathrm{C} /{ }^{13} \mathrm{C}$ ratios are required to improve the statistics, together with multiple $\mathrm{CO}$ transitions to constrain radiative transfer models. Because of the higher kinetic temperatures in PNe, observations in the sub-mm regime may be more useful for this purpose.

A significant effort has been made to detect $\mathrm{CO}$ toward the six $\mathrm{PNe}$ in which ${ }^{3} \mathrm{He}$ have been measured to directly establish the relationship between non-standard mixing in lowmass stars and the ${ }^{3} \mathrm{He}$ and ${ }^{12} \mathrm{C} /{ }^{13} \mathrm{C}$ abundances (PBSTG). The stellar models predict that 
non-standard mixing $(\mathrm{CBP})$ will lower both ${ }^{3} \mathrm{He} / \mathrm{H}$ and ${ }^{12} \mathrm{C} /{ }^{13} \mathrm{C}$. Based on chemical evolution models more than $90 \%$ of low-mass stars must undergo CBP to reconcile the observations of ${ }^{3} \mathrm{He}$ in $\mathrm{H}$ II regions (Charbonnel et al. 1998). Only one object with a measured ${ }^{3} \mathrm{He} / \mathrm{H}$ ratio, NGC 6720, has been detected in CO. Since the measured ${ }^{3} \mathrm{He}$ upper limit of ${ }^{3} \mathrm{He} / \mathrm{H}$ $=0.54 \pm 0.29 \times 10^{-3}$ is consistent with both the standard model and with models which include CBP, the current data do not provide any interesting constraints. Note that the progenitor mass of NGC 6720 is slightly above $2 M_{\odot}$ which is where CBP should turn off.

$\mathrm{CO}$ is typically detected in young PNe where the nebula has not evolved sufficiently to dissociate the molecular material. In contrast, ${ }^{3} \mathrm{He}$ is best detected in evolved, older PNe which tend to fill the telescope's beam. Therefore it is not a coincidence that there is little overlap between the two samples. As pointed out by PBSTG, observations using the CIII] transition toward PNe will have a better opportunity to match the ${ }^{3} \mathrm{He}$ samples. Currently, the number of PNe with either CIII] or ${ }^{3} \mathrm{He}$ detections is sparse. The Green Bank Telescope will offer a significant improvement in our ability to detect ${ }^{3} \mathrm{He}^{+}$in PNe and thus increase the current sample of one well determined ratio (NGC 3242) and two probable detections. Observations of CO in PNe will complement any CIII] data. Because of the large uncertainties in estimating the progenitor masses we feel that the number of detections must be significantly increased to build a strong observational case for non-standard mixing in PNe. It is interesting to note that even if CBP occurs in most low-mass stars it will not be a significant factor in the chemical evolution of ${ }^{12} \mathrm{C} /{ }^{13} \mathrm{C}$ in the Galaxy since the production of carbon is dominated by intermediate-mass stars (PBSTG). Therefore, measurements of ${ }^{3} \mathrm{He}$ is one of the few methods to probe how low-mass stars influence the chemical evolution of the Galaxy.

\section{Summary}

The following are the main results of the $\mathrm{J}=2 \rightarrow 1$ and $\mathrm{J}=3 \rightarrow 2$ millimeter transitions of ${ }^{12} \mathrm{CO}$ and ${ }^{13} \mathrm{CO}$ in 11 Galactic planetary nebulae (PNe).

1. We have detected both ${ }^{12} \mathrm{CO}$ and ${ }^{13} \mathrm{CO}$ emission in 9 PNe for the $\mathrm{J}=2 \rightarrow 1$ transition using the NRAO $12 \mathrm{~m}$ telescope and in 4 PNe for the $\mathrm{J}=3 \rightarrow 2$ transition using the HHT $10 \mathrm{~m}$ telescope. Assuming ${ }^{12} \mathrm{C} /{ }^{13} \mathrm{C}={ }^{12} \mathrm{CO} /{ }^{13} \mathrm{CO}$ the ratios range from $2.2-31$. These results support an extra mixing process in at least some stars.

2. Large velocity gradient (LVG) models have been applied to the 4 PNe where both transitions have been detected. These models were primarily used to determine if the ${ }^{12} \mathrm{CO}$ line is optically thick, an effect which would overestimate the ${ }^{12} \mathrm{C} /{ }^{13} \mathrm{C}$ ratio. A very low 
${ }^{12} \mathrm{C} /{ }^{13} \mathrm{C}$ ratio of 2.2 was determined for M1-16 which does not appear to be a lower limit.

3. The $\mathrm{J}=3 \rightarrow 2$ transition is significantly more intense than the $\mathrm{J}=2 \rightarrow 1$ transition. This result has been confirmed by models using typical densities measured in PNe. This suggests that $\mathrm{CO}$ observations in PN may be better carried out in the sub-millimeter regime.

We thank the technical support staff at both the NRAO $12 \mathrm{~m}$ telescope and the SMTO. In particular, we thank Harold Butner for help during our observations at the HHT. We acknowledge fruitful discussions with Corinne Charbonnel and Bob Rood about stellar evo-

lution. Lastly, we thank Daniele Galli for communicating his results before publication.

\section{REFERENCES}

Abia, C. \& Isern, J. 1997, MNRAS, 289, L11

Bachiller, R., Forveille, T., Huggins, P.J., \& Cox, P. 1997, A\&A, 324, 1123

Bachiller, R., Planesas, P., Martin-Pintado, J., Bujarrabal, V., \& Tafalla, M. 1989, A\&A, 210,366

Balick, B. 1987, AJ, 94, 671

Balser, D. S., Bania, T. M., Rood, R. T., \& Wilson, T. L. 1997, ApJ, 483, 320

Balser, D. S., Bania, T. M., Rood, R. T., \& Wilson, T. L. 1999, ApJ, 510, 759

Balser, D. S., Rood, R. T., \& Bania, T. M. 1999, ApJ, 522, L73

Bania, T. M., Rood, R. T., \& Balser, D. S. 2002, Nature, 415, 54

Boothroyd, A., \& Sackmann, I.-J. 1999, ApJ, 510, 232

Charbonnel, C. 1994, A\&A, 282, 811

Charbonnel, C. 1995, ApJ, 453, L41

Charbonnel, C., Brown, J.A., \& Wallerstein, G. 1998, A\&A, 332, 204

Charbonnel, C., \& Do Nascimento, J. D., Jr. 1998, A\&A, 336, 915

Clegg, R.E.S., Storey, P.J., Walsh, J.R., \& Neale, L. 1997, MNRAS, 284, 348

Cox, P., Omont, A., Huggins, P. J., Bachiller, R., \& Forveille, T. 1992, A\&A, 266, 420 
Dearborn, D.S.P., Schramm, D.N., \& Steigman, G. 1986, ApJ, 302, 35

Dearborn, D.S.P., Steigman, G., \& Tosi, M. 1996, ApJ, 465, 887

Fields, B. D. 1996, ApJ, 456, 478

Forestini, M., \& Charbonnel, C. 1997, A\&AS, 123, 241

Frost, C.A., Cannon, R.C., Lattanzio, J.C., Wood, P.R., \& Forestini, M. 1998, A\&A, 332, L17

Galli, D., Stanghellini, L., Tosi, M., Palla, F. 1997, ApJ, 477, 218

Geiss, J. 1993, in Origin and Evolution of the Elements, ed. N. Prantzos, E. Vangioni-Flam, and M. Casse (Cambridge: Cambridge University Press), 89

Gloeckler, G. \& Geiss, J. 1996, Nature, 381, 210

Gilroy, K.K. 1989, ApJ, 347, 835

Gilroy, K.K. \& Brown, J.A. 1991, ApJ, 371, 578

Górny, S. K., Stasińska, G., \& Tylenda R. 1997, A\&A, 318, 256

Gratton, R. G., Sneden, C., Carretta, E., \& Bragaglia, A. 2000, A\&A, 354, 169

Greaves, J. S., \& Holland, W. S. 1997, A\&A, 327, 342

Herwig, F. 1996, in Stellar Evolution: what should be done, 32nd Liège Int. Astroph. Coll., eds. A. Noels \& N. Grevesse (Univ. Liège), 441

Huggins, P.J., Bachiller, R., Cox, P., \& Forveille, T. 1996, A\&A, 315, 284

Huggins, P.J. \& Healy, A.P. 1989, ApJ, 346, 201

Iben, I. 1964, ApJ, 140, 1631

Iben, I. 1967, ApJ, 147, 624

Iben, I., \& Renzini, A. 1984, Phys. Letters, 105, 329

Jaminet, P. A., Danchi, W. C., Sutton, E. C., Russel, A. P. G., Sandell, G., Bieging, J. H., \& Wilner, D. 1991, ApJ, 380, 461

Kahane, C., \& Jura, M. 1994, A\&A, 290, 183 
Kutner, M. L., \& Ulich, B. L. 1981, ApJ, 250, 341

Lambert, D. L., Gustafsson, B., Eriksson, K, \& Hinkle, K. H. 1986, ApJS, 62, 373

de Laverny, P. \& Gustafsson, B. 1998, A\&A, 332, 661

de Laverny, P. \& Gustafsson, B. 1999, A\&A, 346, 520

Likkel L., Morris, M., Forveille, T., \& Omont, A. 1988, A\&A, 198, L1

Leung, C. M. \& Liszt, H. S. 1976, ApJ, 208, 732

Manchado, A., Guerrero, M. A., Stanghellini, L., \& Serra-Ricart, M. 1996, The IAC Morphological Catalog of Northern Galactic Planetary Nebulae (Tenerife:IAC)

Mangum, J. G. 1999, User's Manual for the NRAO $12 \mathrm{~m}$ Millimeter-Wave Telescope

Miskey, C. L., Feibelman, W. A., \& Bruhweiler, F. C. 2000, BAAS, 32, 743

Mundy, L.G. 1992, http://bima.astro.umd.edu/miriad/intro.html

Ohnaka, K. \& Tsuji, T. 1996, A\&A, 310, 933

Ohnaka, K. \& Tsuji, T. 1998, A\&A, 335, 1018

Ohnaka, K., Tsuji, T., \& Aiki, W. 2000, A\&A, 353, 528

Palla, F., Bachiller, R., Stanghellini, L., Tosi, M., \& Galli, D. 2000, A\&A, 355, 69 (PBSTG)

Prantzos, N. 1996, A\&A, 310, 106

Rood, R. T. 1972, ApJ, 177, 681

Rood, R. T., Bania, T. M., \& Wilson, T. L. 1992, Nature, 355, 618

Rood, R. T., Steigman, G., \& Tinsley, B. M. 1976, ApJ, 207, L57

Sackmann, I.-J., \& Boothroyd, A. 1999, ApJ, 510, 217

Scully, S. T., Cassë, M., Olive, K. A., Schramm, D. N., Truran, J., \& Vangioni-Flam, E. 1996, ApJ, 462, 960

Sobolev, V.V. 1958, Theoretical Astrophysics, ed. V.A. Ambartsumyan Pergamon Press Ltd., London, Chapter 29

Sobolev V.V. 1960, Moving Envelopes of Stars, Harvard University Press 
Stasińska, G., Górny, S. K., \& Tylenda R. 1997, A\&A, 327, 736

Steigman, G., \& Tosi, M. 1992, ApJ, 401, 150

Steigman, G., \& Tosi, M. 1995, ApJ, 453, 173

Sweigart, A. V., \& Mengel, J. G. 1979, ApJ, 229, 624

Tomkin, J., Lambert, D. L., \& Luck, R. E. 1975, ApJ, 199, 436

Tomkin, J., Luck, R. E., Lambert, D. L. 1976, ApJ, 210, 694

Vangioni-Flam, E., Olive, K. A., \& Prantzos, N. 1994, ApJ, 427, 618

Vassiliadis, E., \& Wood, P. R. 1993, ApJ, 413, 641

Wannier, P.G. \& Sahai, R. 1987, ApJ, 319, 367

Wasserburg, G.J., Boothroyd, A., \& Sackmann, I.-J. 1995, ApJ, 447, L37

Weiss, A., Wagenhuber, J., \& Denissenkov 1996, A\&A, 313, 581

Wilson, T. L., Muders, D., Butner, H. M., Gensheimer, P. D., Uchida, K. I., Kramer, C., Tieftrunk, A. R. 2001, in ASP Conf. Series Vol. 235, Science with the Atacama Large Millimeter Array, ed. A. Wootten (San Francisco:ASP), 257 

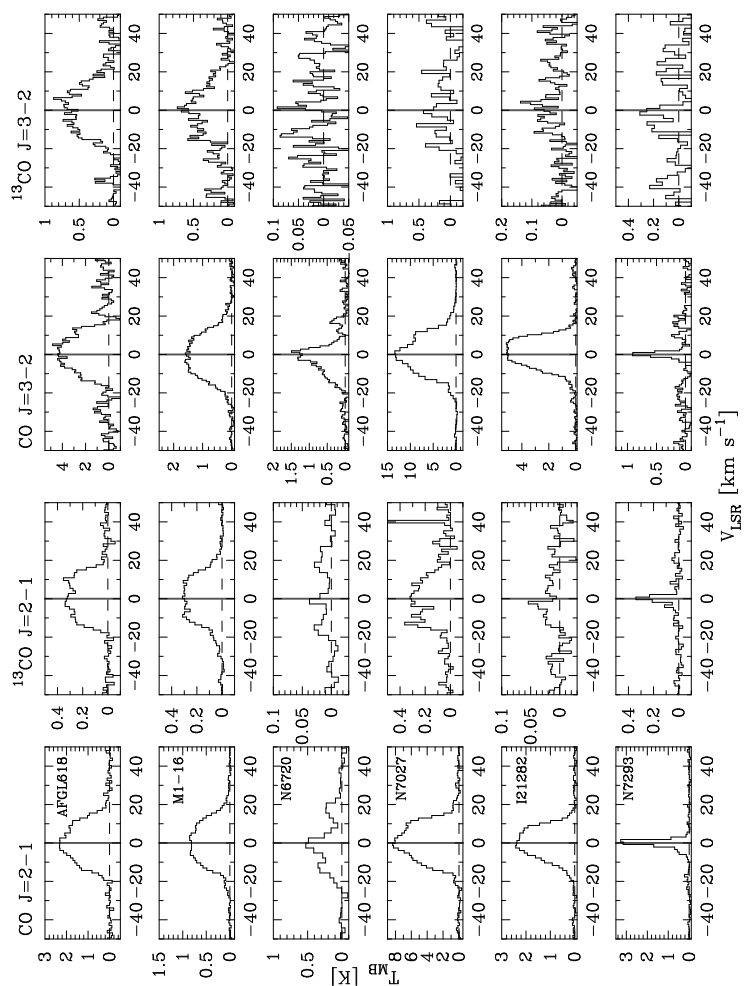
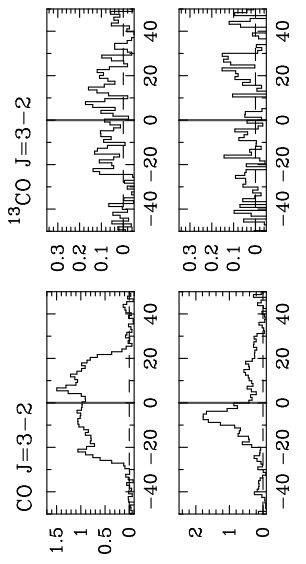

$F_{i s}$

表
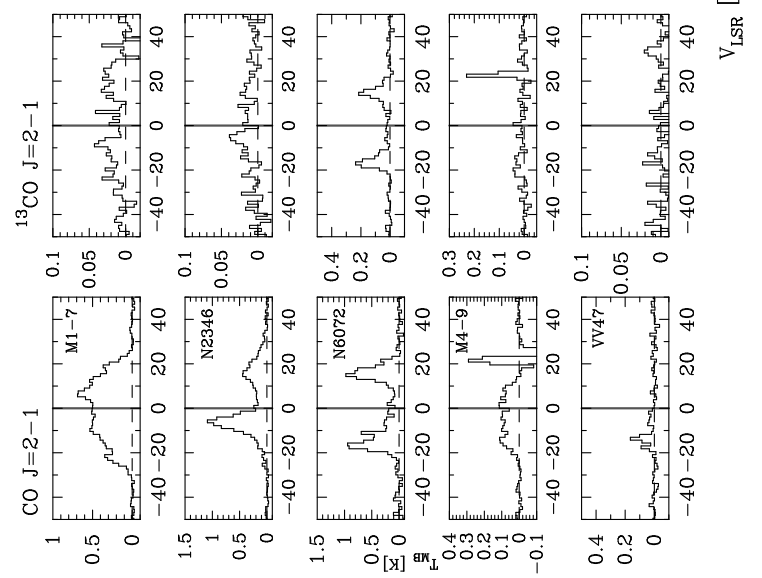

Fig. 1. - NRAO $12 \mathrm{~m}$ spectra of the ${ }^{12} \mathrm{CO}$ and ${ }^{13} \mathrm{CO}$ emission in the $\mathrm{J}=2 \rightarrow 1$ transition (first two columns) and SMTO HHT spectra of the ${ }^{12} \mathrm{CO}$ and ${ }^{13} \mathrm{CO}$ emission in the $\mathrm{J}=3 \rightarrow 2$ transition (last two columns) for our sample of PNe. The intensity scale has been converted into main beam brightness temperature in units of Kelvin. The vertical grey line marks the $V_{\mathrm{LSR}}$ of the source. 


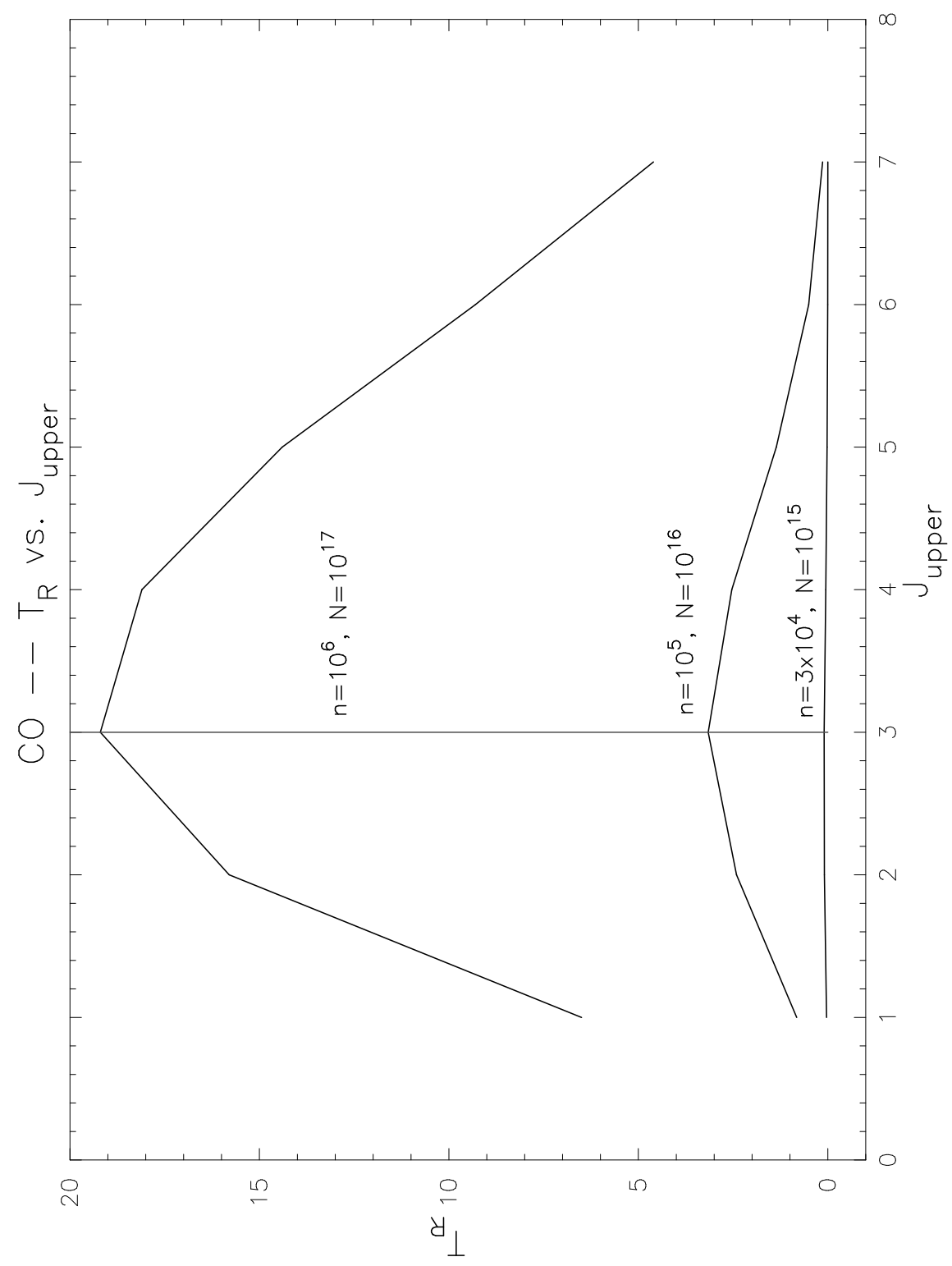

Fig. 2.- Plot of radiation temperature versus lower $\mathrm{J}$ transition over a range of physical conditions. These LVG models indicate that the $\mathrm{J}=3 \rightarrow 2$ line is the peak over the range of values relevant to most planetary nebulae. 


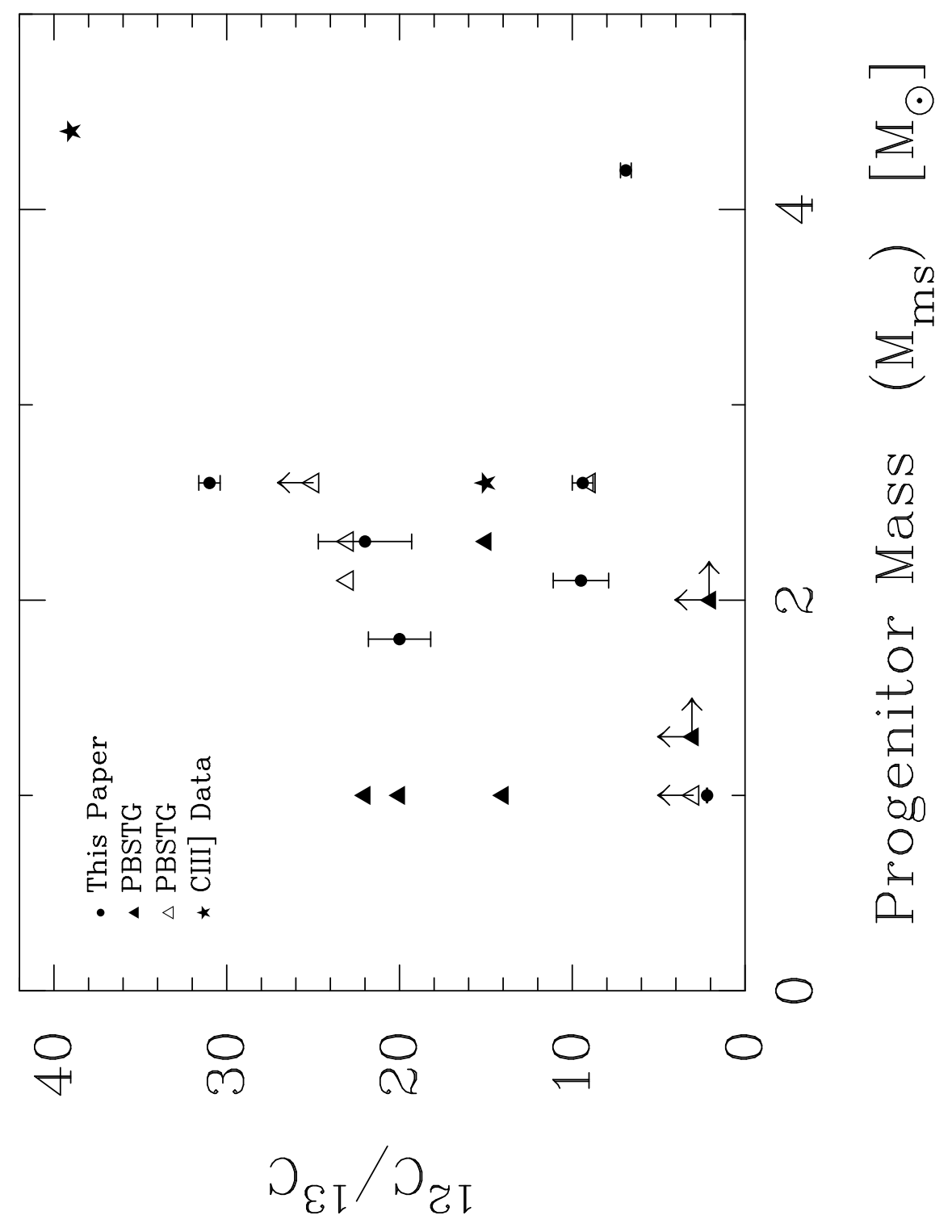

Fig. 3.- The ${ }^{12} \mathrm{C} /{ }^{13} \mathrm{C}$ abundance ratios versus the progenitor main sequence mass. The circles are taken from this paper, the triangles are from PBSTG, and the stars are taken from UV data. The open triangles are from PBSTG and are in common with our sample. 
Table 1. Observed Planetary Nebulae

\begin{tabular}{|c|c|c|c|c|c|c|c|c|}
\hline Name & $\begin{array}{c}(\ell, b) \\
\text { (degrees) }\end{array}$ & $\begin{array}{c}\alpha \\
(1950)\end{array}$ & $\begin{array}{c}\delta \\
(1950)\end{array}$ & $\begin{array}{c}\text { Offset } \\
\text { (arcsec) }\end{array}$ & $\begin{array}{c}V_{\mathrm{LSR}} \\
\left(\mathrm{km} \mathrm{sec}^{-1}\right)\end{array}$ & $\begin{array}{c}\text { Size } \\
(\operatorname{arcsec})\end{array}$ & $\begin{array}{l}M_{\mathrm{ms}} \\
\left(M_{\odot}\right)\end{array}$ & Shape $^{\mathrm{a}}$ \\
\hline AFGL 618 & $(166,-06)$ & 043933.8 & +360115 & $(0,0)$ & -22 & 12 & $\ldots$ & $\mathrm{E}$ \\
\hline M1-7 & $(189,-07)$ & 063418.0 & +240300 & $(0,0)$ & -11 & 11 & 1.8 & $\mathrm{E}$ \\
\hline NGC 2346 & $(215,+03)$ & 070649.6 & -004330 & $(0,0)$ & +9 & 52 & 2.3 & $\mathrm{~B}$ \\
\hline M1-16 & $(226,+05)$ & 073455.4 & -093200 & $(0,0)$ & +50 & $<4$ & 1.0 & B \\
\hline VV 47 & $(164,+31)$ & 075400.0 & +533300 & $(-66,+66)$ & -55 & 380 & $\ldots$ & $\mathrm{E}$ \\
\hline NGC 6072 & $(342,+10)$ & 160941.0 & -360610 & $(0,0)$ & +15 & 70 & 4.2 & B \\
\hline M4-9 & $(024,+05)$ & 181137.4 & -050017 & $(0,+20)$ & -15 & 47 & $\ldots$ & $\ldots$ \\
\hline NGC 6720 & $(063,+13)$ & 185143.7 & +325756 & $(-40,-20)$ & -2.6 & 76 & 2.1 & $\mathrm{E}$ \\
\hline NGC 7027 & $(084,-03)$ & 210509.4 & +420203 & $(0,0)$ & +26 & 15 & 2.6 & $\mathrm{E}$ \\
\hline IRAS 21282 & $(094,-00)$ & 212815.1 & +505047 & $(0,0)$ & +18 & $\ldots$ & $\ldots$ & $\ldots$ \\
\hline NGC 7293 & $(036,-57)$ & 222654.8 & -210541 & $(-372,0)$ & -24 & 660 & 2.6 & $\mathrm{E}$ \\
\hline
\end{tabular}

aplanetary nebulae morphology: $\mathrm{R}=$ Round, $\mathrm{E}=$ Elliptical, and $\mathrm{B}=$ Bipolar. 
Table 2. Observing Log

\begin{tabular}{lcccc}
\hline \hline Telescope & Transition & $\begin{array}{c}\text { Frequency } \\
(\mathrm{GHz})\end{array}$ & $\begin{array}{c}\Theta_{\text {beam }} \\
(\text { arcsec })\end{array}$ & $\begin{array}{c}\Delta v \\
\left(\mathrm{~km} \mathrm{sec}^{-1}\right)\end{array}$ \\
\hline NRAO $12 \mathrm{~m}$ & $\left({ }^{13} \mathrm{CO}\right) \mathrm{J}=2 \rightarrow 1$ & 220.399 & 29 & 1.4 \\
NRAO $12 \mathrm{~m}$ & $\left({ }^{12} \mathrm{CO}\right) \mathrm{J}=2 \rightarrow 1$ & 230.538 & 27 & 1.3 \\
HHT 10 $\mathrm{m}$ & $\left({ }^{13} \mathrm{CO}\right) \mathrm{J}=3 \rightarrow 2$ & 330.588 & 23 & 0.9 \\
HHT $10 \mathrm{~m}$ & $\left({ }^{12} \mathrm{CO}\right) \mathrm{J}=3 \rightarrow 2$ & 345.796 & 22 & 0.9 \\
\hline
\end{tabular}


Table 3. Results

\begin{tabular}{|c|c|c|c|c|c|c|}
\hline Source & Transition & $\begin{array}{c}T_{\text {peak }} \\
(\mathrm{K})\end{array}$ & $\begin{array}{c}R M S \\
(\mathrm{~K})\end{array}$ & $\begin{array}{c}V_{\text {centroid }} \\
\left(\mathrm{km} \mathrm{sec}^{-1}\right)\end{array}$ & $\begin{array}{c}\Delta V^{\mathrm{a}} \\
\left(\mathrm{km} \mathrm{sec}^{-1}\right)\end{array}$ & $\begin{array}{c}I \\
\left(\mathrm{~K} \mathrm{~km} \mathrm{sec}^{-1}\right)\end{array}$ \\
\hline \multirow{4}{*}{ AFGL 618} & ${ }^{12} \mathrm{CO} \mathrm{J}=2 \rightarrow 1$ & 2.4 & 0.09 & -22.0 & 23.9 & $60 . \pm 0.8$ \\
\hline & ${ }^{13} \mathrm{CO} \mathrm{J}=2 \rightarrow 1$ & 0.35 & 0.03 & -20.9 & 25.5 & $8.8 \pm 0.2$ \\
\hline & ${ }^{12} \mathrm{CO} \mathrm{J}=3 \rightarrow 2$ & 5.3 & 0.51 & -21.7 & 22.4 & 120. \pm 4.0 \\
\hline & ${ }^{13} \mathrm{CO} \mathrm{J}=3 \rightarrow 2$ & 0.95 & 0.12 & -20.7 & 27.5 & 23. \pm 0.9 \\
\hline \multirow[t]{4}{*}{ M1-7 } & ${ }^{12} \mathrm{CO} \mathrm{J}=2 \rightarrow 1$ & 0.70 & 0.02 & -10.7 & 35.1 & 22. \pm 0.2 \\
\hline & ${ }^{13} \mathrm{CO} \mathrm{J}=2 \rightarrow 1$ & 0.04 & 0.01 & -10.0 & 53.9 & $1.1 \pm 0.1$ \\
\hline & ${ }^{12} \mathrm{CO} \mathrm{J}=3 \rightarrow 2$ & 1.5 & 0.06 & -11.5 & 39.0 & 49. \pm 0.5 \\
\hline & ${ }^{13} \mathrm{CO} \mathrm{J}=3 \rightarrow 2$ & 0.17 & 0.06 & -4.2 & 45.0 & $3.0 \pm 0.5$ \\
\hline \multirow[t]{4}{*}{ NGC 2346} & ${ }^{12} \mathrm{CO} \mathrm{J}=2 \rightarrow 1$ & 1.1 & 0.02 & 6.0 & $10.1^{\mathrm{b}}$ & 18. \pm 0.2 \\
\hline & ${ }^{13} \mathrm{CO} \mathrm{J}=2 \rightarrow 1$ & 0.04 & 0.01 & 6.6 & $31.8^{\mathrm{b}}$ & $0.81 \pm 0.1$ \\
\hline & ${ }^{12} \mathrm{CO} \mathrm{J}=3 \rightarrow 2$ & 1.8 & 0.13 & 2.3 & 13.0 & 30. \pm 1.3 \\
\hline & ${ }^{13} \mathrm{CO} \mathrm{J}=3 \rightarrow 2$ & $<0.24$ & 0.08 & $\cdots$ & $\cdots$ & $\ldots$ \\
\hline \multirow{4}{*}{ M1-16 } & ${ }^{12} \mathrm{CO} \mathrm{J}=2 \rightarrow 1$ & 0.87 & 0.02 & 50.2 & 25.9 & 25. \pm 0.2 \\
\hline & ${ }^{13} \mathrm{CO} \mathrm{J}=2 \rightarrow 1$ & 0.32 & 0.01 & 50.0 & 28.0 & $9.8 \pm 0.1$ \\
\hline & ${ }^{12} \mathrm{CO} \mathrm{J}=3 \rightarrow 2$ & 1.8 & 0.10 & 49.4 & 26.2 & 49. \pm 0.8 \\
\hline & ${ }^{13} \mathrm{CO} \mathrm{J}=3 \rightarrow 2$ & 0.80 & 0.11 & 48.6 & 31.1 & 21. \pm 1.0 \\
\hline \multirow[t]{2}{*}{ VV 47} & ${ }^{12} \mathrm{CO} \mathrm{J}=2 \rightarrow 1$ & 0.17 & 0.02 & -68.3 & 3.25 & $0.92 \pm 0.1$ \\
\hline & ${ }^{13} \mathrm{CO} \mathrm{J}=2 \rightarrow 1$ & $<0.03$ & 0.01 & $\cdots$ & $\cdots$ & $\ldots$ \\
\hline \multirow[t]{2}{*}{ NGC 6072} & ${ }^{12} \mathrm{CO} \mathrm{J}=2 \rightarrow 1$ & 1.0 & 0.05 & 12.3 & $10.7^{\mathrm{b}}$ & 18. \pm 0.4 \\
\hline & ${ }^{13} \mathrm{CO} \mathrm{J}=2 \rightarrow 1$ & 0.24 & 0.01 & 11.5 & $5.16^{\mathrm{b}}$ & $2.6 \pm 0.1$ \\
\hline \multirow[t]{3}{*}{ M4-9 } & ${ }^{12} \mathrm{CO} \mathrm{J}=2 \rightarrow 1$ & 0.12 & 0.01 & -19.5 & 26.8 & $2.8 \pm 0.1$ \\
\hline & ${ }^{13} \mathrm{CO} \mathrm{J}=2 \rightarrow 1$ & $<0.03$ & 0.01 & $\ldots$ & $\cdots$ & $\ldots$ \\
\hline & ${ }^{12} \mathrm{CO} \mathrm{J}=2 \rightarrow 1$ & 0.54 & 0.02 & -4.0 & 27.0 & 11. \pm 0.4 \\
\hline
\end{tabular}


Table 3-Continued

\begin{tabular}{ccccccc}
\hline \hline Source & Transition & $\begin{array}{c}T_{\text {peak }} \\
(\mathrm{K})\end{array}$ & $\begin{array}{c}R M S \\
(\mathrm{~K})\end{array}$ & $\begin{array}{c}V_{\text {centroid }} \\
\left(\mathrm{km} \mathrm{sec}^{-1}\right)\end{array}$ & $\begin{array}{c}\Delta V^{\mathrm{a}} \\
\left(\mathrm{km} \mathrm{sec}^{-1}\right)\end{array}$ & $\begin{array}{c}I \\
\left(\mathrm{~K} \mathrm{~km} \mathrm{sec}^{-1}\right)\end{array}$ \\
\hline NGC 6720 & ${ }^{13} \mathrm{CO} \mathrm{J}=2 \rightarrow 1$ & 0.05 & 0.01 & -3.5 & 49.1 & $1.2 \pm 0.2$ \\
& ${ }^{12} \mathrm{CO} \mathrm{J}=3 \rightarrow 2$ & 1.4 & 0.11 & -4.7 & 16.3 & $22 . \pm 0.9$ \\
& ${ }^{13} \mathrm{CO} \mathrm{J}=3 \rightarrow 2$ & $<0.12$ & 0.04 & $\cdots$ & $\cdots$ & $\cdots$ \\
& & & & & & \\
NGC 7027 & ${ }^{12} \mathrm{CO} \mathrm{J}=2 \rightarrow 1$ & 8.4 & 0.003 & 26.8 & 22.3 & $200 . \pm 2.0$ \\
& ${ }^{13} \mathrm{CO} \mathrm{J}=2 \rightarrow 1$ & 0.37 & 0.004 & 22.8 & 27.4 & $6.4 \pm 0.9$ \\
& ${ }^{12} \mathrm{CO} \mathrm{J}=3 \rightarrow 2$ & 13. & 0.65 & 26. & 22. & $300 . \pm 2.0$ \\
& ${ }^{13} \mathrm{CO} \mathrm{J}=3 \rightarrow 2$ & $<1.0$ & 0.34 & $\cdots$ & $\cdots$ & $\cdots$ \\
& & & & & & \\
IRAS 21282 & ${ }^{12} \mathrm{CO} \mathrm{J}=2 \rightarrow 1$ & 2.5 & 0.06 & 18.1 & 18.4 & $50 . \pm 0.4$ \\
& ${ }^{13} \mathrm{CO} \mathrm{J}=2 \rightarrow 1$ & 0.06 & 0.01 & 15.6 & 18.8 & $0.66 \pm 0.1$ \\
& ${ }^{12} \mathrm{CO} \mathrm{J}=3 \rightarrow 2$ & 5.1 & 0.13 & 18.9 & 17.3 & $100 . \pm 0.9$ \\
& ${ }^{13} \mathrm{CO} \mathrm{J}=3 \rightarrow 2$ & 0.15 & 0.04 & 21.8 & 19.8 & $1.5 \pm 0.3$ \\
& & & & & & \\
& ${ }^{12} \mathrm{CO} \mathrm{J}=2 \rightarrow 1$ & 3.3 & 0.05 & -15.4 & 3.5 & $15 . \pm 0.2$ \\
NGC 7293 ${ }^{13} \mathrm{CO} \mathrm{J}=2 \rightarrow 1$ & 0.34 & 0.02 & -15.3 & 3.2 & $1.6 \pm 0.1$ \\
& ${ }^{12} \mathrm{CO} \mathrm{J}=3 \rightarrow 2$ & 0.97 & 0.12 & -14.8 & 2.6 & $2.5 \pm 0.2$ \\
& ${ }^{13} \mathrm{CO} \mathrm{J}=3 \rightarrow 2$ & 0.29 & 0.08 & -15.7 & 2.3 & $0.52 \pm 0.2$ \\
\hline
\end{tabular}

aBased on a one-component Gaussian model.

${ }^{b}$ Multiple components exists within the single Gaussian fit. 
Table 4. ${ }^{12} \mathrm{C} /{ }^{13} \mathrm{C}$ Abundance Ratios

\begin{tabular}{lccccc}
\hline \hline & & \multicolumn{4}{c}{${ }^{12} \mathrm{C} /{ }^{13} \mathrm{C}$ Ratio } \\
\cline { 3 - 6 } \multicolumn{1}{c}{ Source } & PK & $\begin{array}{c}\text { NRAO } 12 \mathrm{~m} \\
(\mathrm{~J}=2 \rightarrow 1)^{\mathrm{a}}\end{array}$ & $\begin{array}{c}\text { HHT } 10 \mathrm{~m} \\
(\mathrm{~J}=3 \rightarrow 2)^{\mathrm{a}}\end{array}$ & Adopted $^{\mathrm{b}}$ & PBSTG $^{\mathrm{c}}$ \\
\hline AFGL 618 & $(166-06)$ & $6.8(6.9)$ & $5.2(5.5)$ & $>4.6^{\mathrm{d}}$ & $4^{\mathrm{e}}$ \\
M1-7 & $(189-07)$ & $20 .(18)$. & $>6.7$ & $20 . \pm 1.8$ & $\ldots$ \\
NGC 2346 & $(215+03)$ & $22 .(28)$. & $>6.3$ & $22 . \pm 2.7$ & 23 \\
M1-16 & $(226+05)$ & $2.6(2.7)$ & $2.4(2.2)$ & $2.2 \pm 0.03$ & $3^{\mathrm{e}}$ \\
VV 47 & $(164+31)$ & $>5.7$ & $\ldots$ & $>5.7$ & $\ldots$ \\
NGC 6072 & $(342+10)$ & $6.9(4.2)$ & $\ldots$ & $6.9 \pm 0.31$ & $\ldots$ \\
M4-9 & $(024+05)$ & $>4.0$ & $\ldots$ & $>4.0$ & 18 \\
NGC 6720 & $(063+13)$ & $9.5(10.8)$ & $>14$. & $9.5 \pm 1.6$ & 22 \\
NGC 7027 & $(084-03)$ & $31 .(22)$. & $>13$. & $31 . \pm 0.62$ & $25^{\mathrm{e}}$ \\
IRAS 21282 & $\ldots$ & $76 .(42)$. & $66 .(33)$. & $>32 .{ }^{\mathrm{d}}$ & $\ldots$ \\
NGC 7293 & $(036-57)$ & $9.4(9.7)$ & $4.8(3.3)$ & $9.4 \pm 0.60$ & 9 \\
\hline
\end{tabular}

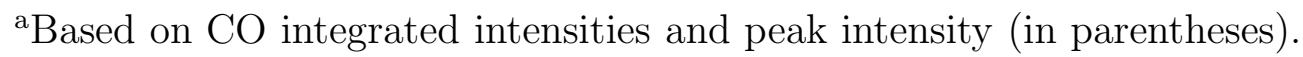

${ }^{\mathrm{b}}$ Adopted ${ }^{12} \mathrm{C} /{ }^{13} \mathrm{C}$ ratio (see text).

${ }^{\mathrm{c}}$ From Palla et al. (2000).

${ }^{\mathrm{d}}$ Based on LVG model.

${ }^{\mathrm{e}}$ Lower limit based on optically thick CO. 